\title{
Elfenbein als Nasenimplantat bei Jacques Joseph
}

\author{
Spätergebnisse nach über 40 Jahren \\ O. Staindl ${ }^{1}$, S. Hellmich ${ }^{2}$, A. Berghaus ${ }^{3}$ \\ ${ }^{1}$ Hals-Nasen-Ohren-Abteilung der Landeskrankenanstalten Salzburg (Vorstand: Prof. Dr. K. Albegger) \\ ${ }^{2}$ HNO-Klinik im St.-Johannes-Hospital Dortmund (Chefarzt: Prof. Dr. S. Hellmich) \\ ${ }^{3}$ HNO-Klinik im Klinikum Steglitz der Freien Universität Berlin (Leiter: Prof. Dr. H. Scherer)
}

\section{Zusammenfassung}

Aufgrund schlechter Erfahrungen mit der Resorption von Knochentransplantaten benutzte Jacques Joseph in geeigneten Fällen Elfenbeinspäne für die Sattelnasenkorrektur. Vor dem Hintergrund, daß sicher nicht mehr viele von Joseph so operierte Patienten am Leben sind, berichten die Autoren über Langzeitergebnisse von 3 Fällen, bei denen 42, 43 und 63 Jahre zuvor Elfenbeinspäne in den Nasenrücken implantiert worden waren. Die Langzeitprognose aus heutiger Sicht und Josephs eigene Beobachtungen zu diesem Material sowie einige auch heute noch nicht allseits geklärte Umstände des Wirkens dieses genialen Operateurs werden besprochen.

\section{Jacques Joseph's Ivory Implants for Rhinoplasty. Late Results after more than 40 Years}

A report is represented on late results of saddle-nose corrections in three patients who, more than 40 years ago, underwent surgery performed by Jacques Joseph in Berlin using an ivory chip. One patient showed a very good permanent result, while the two others had implant loss, which, in one case, was due to trauma. The case reports offer an opportunity to recall a few biographical data on Jacques Joseph and on his use of ivory as an implant material.

\section{Einleitung}

Die Sattelbildung ist ein häufiger Formfehler der Nase. Bei Vorliegen einer echten Einsattelung ist ein grundlegendes Bestreben der Korrektur immer der Ersatz fehlender Strukturen durch geeignetes Stütz- und Füllmaterial. Daher kommen bei Sattelnasen häufig Transplantate oder Spanimplantate zum Einsatz. Das verwendete Material spielt dabei eine entscheidende Rolle, weil von seinem postoperativen Verhalten die Dauerhaftigkeit des primär erzielten Operationsergebnisses mitbestimmt wird. Das Schicksal des Implantates selbst hängt von der Wechsel-

Laryngo-Rhino-Otol. 68 (1989) 576-580

(C) Georg Thieme Verlag Stuttgart - New York wirkung zwischen dem Implantatlager und dem verwendeten Material ab (Hellmich, 1972).

Die Liste der Werkstoffe, die bereits zum Wiederaufbau der menschlichen Nase verwendet worden sind, erscheint endlos. Ob es sich um Teile patienteneigener Zehen oder Finger handelte, die zunächst gestielt in die Nase eingenäht und später abgetrennt wurden, ob es Fingernägel, Brustbeine von Enten, verschiedene Steinarten, Pflanzenteile, Gold, Silber, Porzellan, gehärtetes Paraffin oder Kunststoffe wie Silikon, Teflon oder „kïnstliche Knochen" waren - alle diese Materialien haben sich langfristig nicht bewährt (Hellmich, 1979; Staindl, 1983).

Ein Implantationsmaterial, welches sich über längere Zeiträume besonderer Beliebtheit erfreute, war Elfenbein. Es wurde als Füllmaterial bei verschiedenen Gesichtsdefekten, insbesondere aber zum Aufbau der Profillinie bei Sattelnasen vor allem von Jacques Joseph verwendet. Joseph wurde am 6.9. 1865 in Königsberg geboren und ist am 12.2. 1934 in Berlin verstorben. Er war nicht nur in einer Privatpraxis tätig, sondern auch von 1916 bis 1921 Leiter der "Abteilung für Gesichtsplastik“ an der von Geheimrat Passow gefuihrten Ohren- und $\mathrm{Na}$ senklinik der Charité in Berlin. Joseph schätzte die Unterbringung in der Ohren-Nasenklinik, weil häufig der plastischen Gesichtsoperation die Sanierung verletzter oder entzündeter Höhlen des Gesichtsschädels vorausging (Reintanz, 1985). Heute leben wohl nur noch wenige Patienten dieses genialen Chirurgen, der primär Orthopäde war, in seiner Todesanzeige aber als „Facharzt für Nasen- und Gesichtsplastik“ bezeichnet wurde (Natvig, 1982; Reintanz, 1985).

Dem Zufall ist es zu verdanken, daß wir über drei Fälle von Sattelnasenkorrekturen unter Verwendung von Elfenbein durch Jacques Joseph berichten können.

\section{Jacques Joseph und das Elfenbein}

Joseph hat den Einsatz von Elfenbeinspänen in seinem Lehrbuch „Nasenplastik und sonstige Gesichtsplastik" angegeben, welches wohl hinsichtlich sprachlicher Klarheit, didaktischer Úbersicht und Dokumentation zu den Meisterwerken unter den medizinischen Lehrbüchern gezählt werden kann. Im folgenden sind Originalzitate apostrophiert, die Seitenangaben beziehen sich auf die Ausgabe dieses Buches im Verlag Curt Kabitzsch, Leipzig, aus dem Jahre 1931 (Joseph, 1931). 
Joseph schreibt: „1915 empfiehlt Eitner (Eitner, 1915) für die Korrektur der Sattelnase Elfenbein. Ungefähr zur selben Zeit verwandte $J$. Joseph, angeregt durch Th. Gluck, der bekanntlich das Elfenbein in der Chirurgie vielfach vorher angewandt hat (Fußnote auf Seite 240 ohne Literaturangabe), bei Sattelnase das Elfenbein statt des Tibiastückes, weil Elfenbein viel weniger resorbiert wird als der eigene lebende Knochen vom Patienten. Aus dem selben Grund hat J. Joseph zur Verbesserung, d. h. Erhöhung der Nasenspitze statt des Lexerschen Knochenstückchens oft Elfenbein eingefügt in Fällen, in denen nicht genügend dicker Ohrknorpel zur Verfügung stand. Ich selbst kann bestätigen, daß ein zur Korrektur der Sattelnase eingefügtes Elfenbeinstück nach neun Jahren in unverändertem Zustand geblieben ist" (Seite 240).

Ein eigenes Kapitel behandelt die „Einfügung von Elfenbein, Paraffin und sonstigem leblosen Material": „Statt lebendiger Knochen oder Knorpelstücke können auch Elfenbein, Paraffin, Bernstein, Gold, Silber, usw. eingefügt werden (Alloplastik). Die letztgenannten dürften nur ganz selten Anwendung finden. Am häufigsten kommt die Verwendung von Elfenbein und Paraffin in Betracht.“... „Elfenbein und sonstiges totes Material werden natülich gleichfalls sterilisiert und nach Herstellung der passenden Form in der selben Weise unter die Haut eingefügt wie der Knochen" (Seite 61).

Eine Operationsaufnahme und eine Operationsskizze (Figur 116 und 117, Seite 63 und 64) demonstrieren die intranasale Einfügung eines Knochenstückes nach $J$. Joseph. Figur 532 (Seite 280) zeigt ein Elfenbeinstück im Röntgenbild, welches bei einer totalen Nasendefektrekonstruktion eingefügt wurde. Auf Seite 332 schreibt Joseph: „Seit etwa 10 Jahren habe ich auf Anregung von Gluck, der bekanntlich das Elfenbein zum Ersatz von Knochendefekten an Kiefern und an den Extremitäten angewandt hat, auch dieses sehr modellierbare Material bei der Korrektur von Sattelnasen und auch in der totalen Rhinoneoplastik angewandt und zwar in der Regel mit sehr gutem Erfolg“.

Es wird auch über Komplikationen berichtet: „Kommt ein eingepflanzter Knorpel zur Vereiterung, so muß er am besten bald entfernt werden. Dagegen kann man ein vereitertes Elfenbeinstück nach Inzision der Haut ruhig an Ort und Stelle liegen lassen. Die Höhle wird durch mehrmaliges Einblasen von Jodoformpulver gereinigt. Die Wunde heilt dann meist per secundam, ohne daß die Gestalt verschlechtert wird " (Seite 337).

Ein eigener Absatz behandelt das „Durchspießen der vorderen Knochen- resp. Elfenbeinspitze": "Natürlich ist es notwendig, auch in Einzelheiten technisch richtig zu operieren. Hierzu rechne ich - und möchte diesen Punkt betonen - daß das vorderste die Nasenspitze bildende Ende des dorsalen Knochenstückes ein wenig mit der Feile abgerundet wird, da es sonst leicht nachträglich die Haut durchspießt und so nach anfänglich guter Einheilung sekundär eine partielle Nekrose des implantierten Knochens eintreten kann. Stellt eine solche Komplikation auch den endgültigen Erfolg nicht in Frage, so erfordert sie doch Nachoperationen und verlängert so erheblich die Behandlungsdauer. Die dauernde Einheilung des Nasodor- salknochens kann ferner noch dadurch gesichert werden, daß man seine vorderste Partie einige Millimeter entfernt von der Hautoberfläche in die Fettschicht einbettet" (Seite 337).

Beim ,indirekten Nasenspitzenersatz aus der Stirnhaut" wurde ebenfalls Elfenbein verwendet: „Figur 657 zeigt die Methode in einem Fall von totalem Nasendefekt. Figur 659 zeigt den in diesem Fall erreichten Enderfolg, zu dem auch die Verschmälerung und Einfügung einer Elfenbeinleiste beigetragen hat. Diese Methode stellt eine Fortsetzung der frontalen Rhinoneoplastik bei Nasendefekten mit fehlendem Skelett dar" (Seite 398).

Zur Rekonstruktion des Septums scheint sich das Elfenbein jedoch nicht bewährt zu haben: „Für die Septumbildung verwende ich stets ein Tibiastuick, das beim Erwachsenen sich mit der Haut fest verfilzt, und nicht Elfenbein, das keine so innige Verbindung mit der Nachbarschaft eingeht" (Seite 471). Gute Resultate wurden mit Elfenbein wohl nur bei der Verwendung am Nasenrücken erzielt: „Die Figuren 894 und 895 betreffen eine Patientin, bei der im Alter von 12 Jahren das Elfenbein eingefügt worden ist. Figur 896 zeigt den Zustand im Alter von 21 Jahren und Figur 897 das in diesem Alter, also 9 Jahre nach der Operation, aufgenommene Röntgenbild" (Seite 433).

Ein Absatz auf Seite 424 gibt auch über die Gründe für die Bevorzugung des Elfenbeins und dessen Behandlung Auskunft: „In letzter Zeit habe ich - mit Rücksicht auf die Erfahrungen, daß die implantierten Tibiastükke zuweilen schon nach einigen Monaten eine teilweise Resorption im Sinne der Abmagerung zeigen - in geeigneten Fällen Elfenbeinstücke eingefügt. Diese hielten nach meinen in letzter Zeit gemachten Beobachtungen der Resorption länger stand als die Tibiastücke. Die sichere Sterilisation des Elfenbeines erfordert nach $B a b(1)$, der auf meine Anregung sich mit dieser Frage beschäftigte, bei infiziertem Material nur 8 Minuten; zur Vorsicht verwende ich dazu 20 bis 30 Minuten". Als Desinfektionsmittel wurden Alkohol und Sublimat benutzt.

In vielen Abbildungen seines Buches (Figur 876 bis 881,887 und 888,894 bis 897 ) ist dokumentiert, daß Joseph mit der Implantation von Elfenbeinspänen in den Nasenrücken hervorragende Resultate bei Sattelnasenkorrekturen erzielen konnte.

Die folgenden Verlaufsbeobachtungen aus den Jahren 1972 bis 1982 zeigen, daß auch über 40 Jahre postoperativ noch ein Spektrum an Langzeitresultaten möglich ist, das von der Implantatabstoßung bis zur reizlosen Inkorporierung des Elfenbeins reicht.

\section{Fallbericht Nr. 1 (Staindl)}

1972 wurde eine damals 63jährige Patientin an der Hals-Nasen-Ohren-Abteilung der Landeskrankenanstalten Salzburg stationär aufgenommen. Sie wies eine eher geringgradige, entzündliche Schwellung der gesamten Nase auf. Darüber hinaus bestand am Nasenrücken ein kleiner Defekt, der von Granulationsgewebe umgeben war. In der Tiefe dieses Defektes war ein gelblich verfärbter Fremdkörper zu sehen und zu tasten. Dieser erwies sich nach Entfernung als ein an 5 Stellen perforiertes, $4,5 \times 2 \mathrm{~cm}$ messendes 


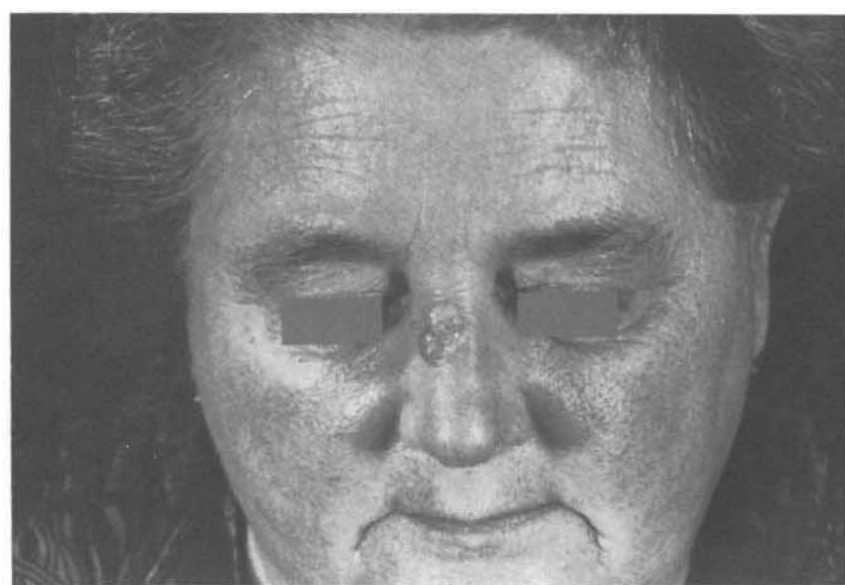

Abb.1 63jährige Patientin mit einem am Nasenrücken perforierten Elfenbeinimplantat.

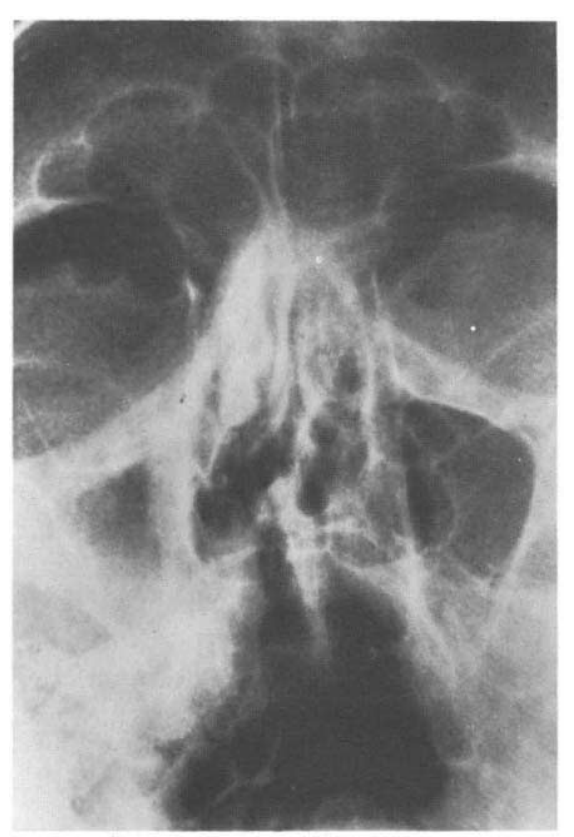

Abb.3 Röntgenbild eines nach rechts abgerutschten Elfenbeinspans in situ a.p.

Elfenbeinstück. Anamnestisch gab die Patientin an, daß ihr dieses Implantat 1929 in Berlin von einem „sehr berühmten Chirurgen“, an dessen Namen sie sich zunächst nicht erinnern konnte, eingepflanzt worden sei. Auf die Frage, ob dieser Chirurg Prof. Joseph war, hat die Patientin dies ausdrücklich bestätigt. Das Elfenbeinstück war also 43 Jahre reizlos im Nasenrücken gelegen. Die Perforation des Fremdkörpers war nach einer Verletzung der äußeren Haut anläßlich eines Sturzes aufgetreten. Hätte sich die Patientin zum damaligen Zeitpunkt nicht an der Nase verletzt, wäre das Implantat vermutlich noch heute reizlos in situ (Abb. 1 und 2).

\section{Fallbericht Nr. 2 (Hellmich)}

1972 kam ein 66 jähriger Patient in der Abteilung für HNO-Krankheiten der Medizinischen Fakultät der RWTH Aachen zur stationären Aufnahme. Anamnestisch ergab sich eine 1930 durch J. Joseph in Berlin zum Ausgleich einer posttraumatischen Sattelnase durchgeführte Spanimplantation in den Nasenrük-

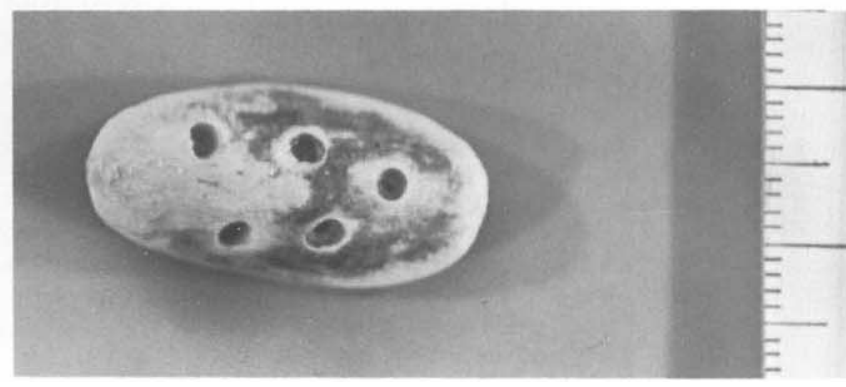

Abb. 2 Das bei der Patientin aus Abb. 1 entnommene Elfenbeinimplantat (Fallbericht Staindl).

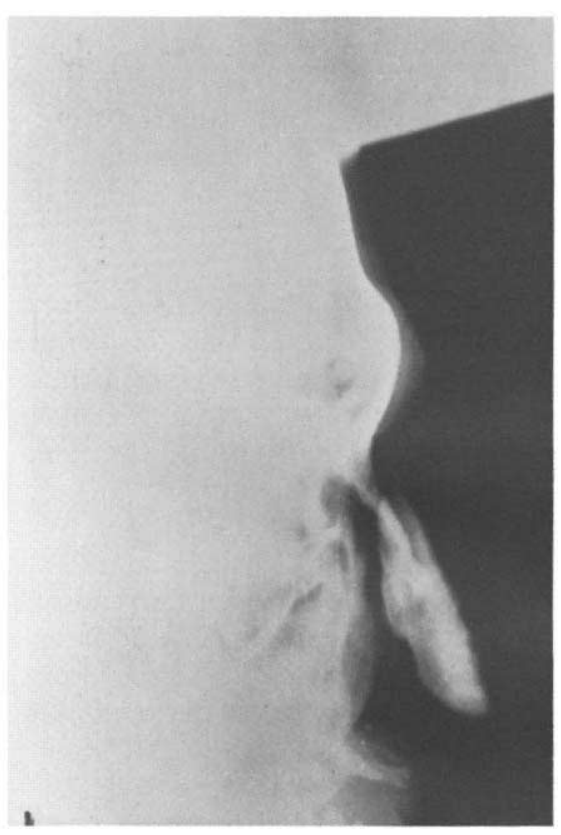

Abb. 4 Seitliche Röntgenaufnahme des Spans vor der Entfernung (Fallbericht Hellmich). Der Span ist in der Form ähnlich dem in Figur 532 des Buches von Joseph abgebildeten Implantat.

ken. Nach Angaben des Patienten war es bereits ein Jahr später durch „Abwandern" des Spans zu einer Schiefnase nach rechts und zur erneuten Sattelbildung gekommen. Der Span sei mobil geblieben und die Härte des Materials habe gestört. Während des Krieges seien diese Probleme unbeachtet geblieben. In der Zeit nach 1945 seien jedoch zusätzlich mehrfache Entzündungen auf dem Nasenrücken und in der Nasenspitze (über dem Span und an dessen kaudalem Ende) aufgetreten. Zur Zeit der stationären Aufnahme im Jahr 1972 bestanden diese Beschwerden erneut und es drohte die Abstoßung des Implantates. Es erfolgte deshalb über einen Hemitransfixionsschnitt rechts die Entfernung des Spans aus seinem nur gering eingekapselten Lager im paramedianen Bereich des Nasenrüickens rechts. Es ergab sich ein solides, gelbliches Elfenbeinimplantat von $4 \times 1,5 \mathrm{~cm}$ Größe. Das entfernte Material wurde in gleicher Sitzung durch einen merthiolat-konservierten Rippenknorpelspan aus der Knorpelbank ersetzt, nachdem auch das destruierte Septum durch einen entsprechenden Septumspan wieder aufgebaut worden war. Der postoperative Verlauf war komplikationslos (Abb. 3 und 4). 


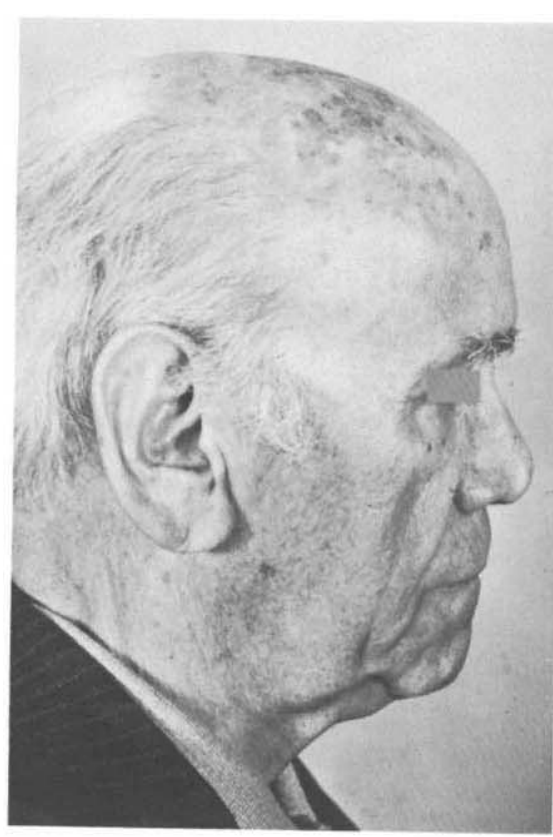

Abb. 5 Patient H. F. Zustand nach Elfenbeinspanimplantation 1919. Profilaufnahme 1982

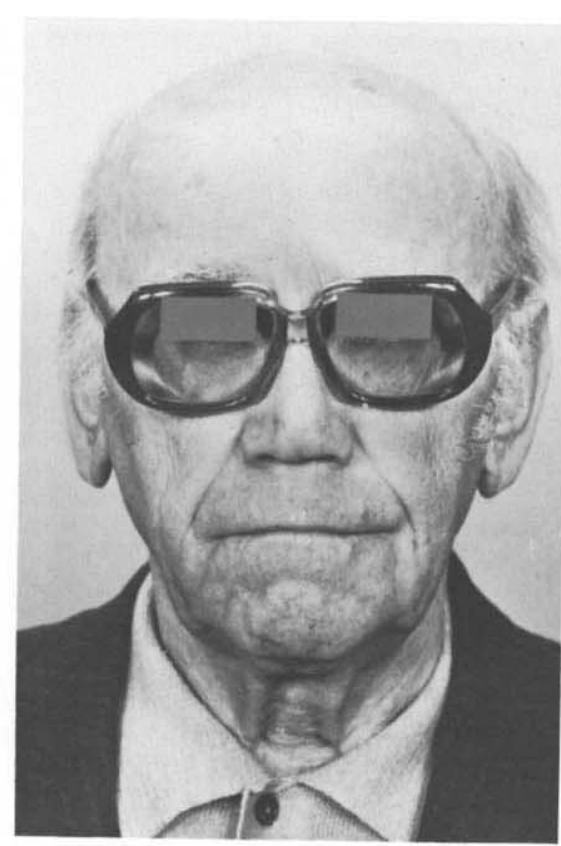

Abb. 6 Patient H. F, en-face-Fotografie mit Brille (1982).

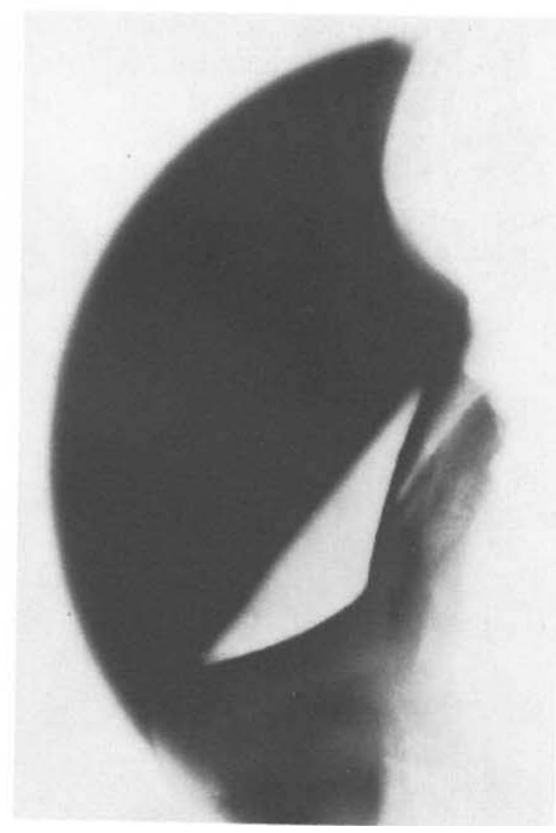

Abb. 7 Seitliche Röntgenaufnahme des Elfenbeinspans des Patienten H. F. (1982). Der Span ist in der Form ähnlich den in Figur $876,878,879$ und 897 des Buches von Joseph abgebildeten Implantaten (Fallbericht Berghaus).

\section{Fallbericht Nr. 3 (Berghaus)}

Der Patient H. F., geboren 25. 1. 1901, stellte sich im April 1982 in der Hals-Nasen-Ohren-Poliklinik des Klinikums Steglitz in Berlin vor. Er klagte über zunehmende Heiserkeit. Die Spiegelung ergab eine Leukoplakie der rechten Stimmlippe, eine später erfolgte Stützautoskopie mit Probeentnahme schloß jedoch einen malignen Prozeß aus.

Herr F. gab anläßlich der Untersuchung auch an, ihm sei im Jahre 1919 an der Charité ein Elfenbeinspan zur Korrektur einer Sattelnase implantiert worden. Nach diesem Eingriff habe er nie irgendwelche Schwierigkeiten mit dem Implantat gehabt und sei auch mit dem Ergebnis des Eingriffes zufrieden. Auf genaueres Befragen glaubte Herr F. sich erinnern zu können, daß der Operateur durchaus "Joseph" geheißen haben könnte. Die Palpation ließ einen reizlos im Nasenrücken eingeheilten Span erkennen, der etwas beweglich war. Die Nasenhaupthöhlen waren unauffällig und reizfrei. Von besonderem Interesse ist in diesem Zusammenhang, daß Herr F. bereits seit vielen Jahren eine Brille trug. Er hatte sich für ein nicht eben leichtgewichtiges Gestell entschieden, welches den Elfenbeinspan wohl nicht unwesentlich belastet hat (Abb. 5 und 6). Herr F. gestattete uns die Anfertigung einer seitlichen Röntgenaufnahme, die den Span in seiner typischen Konfiguration und Lage mühelos identifizierbar machte (Abb. 7). Der Patient blieb nach der erfolgten Stützautoskopie noch einige Zeit in unserer Nachbetreuung und wurde später noch einmal wegen internistischer Probleme in der Medizinischen Klinik des Klinikums Steglitz behandelt. Solange wir Kontakt zu ihm hatten, traten keinerlei Probleme mit dem Nasenimplantat auf.

Die 3 Fälle bestätigen, daß für die besonderen Belange der Nase und des Nasenrükkens zu harte und unelastische Implantate - und darum handelt es sich bei Elfenbein - in Einzelfällen ihren Zweck durchaus erfüllen können, in der Mehrzahl aber langfristig perforieren und schon deshalb entfernt werden müssen. Ganze Sammlungen entfernter entsprechender Implantate - auch und gerade von Elfenbeinspänen - geben hierzu ein beredtes Zeugnis.

\section{Diskussion}

Bei dem 1919 an der Charité operierten Patienten (H. F., Fallbericht Berghaus) kann mit hinreichender Wahrscheinlichkeit davon ausgegangen werden, daß er tatsächlich von Jacques Joseph operiert wurde; denn der Operationstermin fällt genau in die Zeit, in der Joseph an der Ohren- und Nasenklinik der Charité seine Abteilung für Gesichtsplastik leitete. Es ist nicht bekannt, daß dort andere außer ihm zu dieser Zeit Elfenbeinimplantate verwendet hätten. Auch die in den Fallberichten Nr. 1 und Nr. 2 (Staindl, Hellmich) erwähnten Patienten sind wohl von Joseph operiert worden, denn sie gaben dies selbst an.

Es ist erstaunlich, wie weit $J$. Joseph als Orthopäde und Nicht-Rhinologe die Septorhinoplastik im modernen Sinn bereits entwickelt hat. Sicher ist es eines seiner Verdienste, damit für den Bereich der Otorhinolaryngologie und seine regionale, fachbezogene plastische Chirurgie zukunftsweisende Pionierarbeit geleistet zu haben.

Jacques Joseph hat Elfenbeinimplantate nicht nur zum Aufbau von Sattelnasen verwendet, sondern berichtet auch über eine ganze Reihe weiterer Indikationen zum Einsatz dieses Materials. Es wurde zur Augmentation am Oberkiefer, Unterkiefer, Jochbein, Stirnbein, in den 
Wangen und zum Ohrmuschelaufbau benutzt. Zumindest bei der Ohrmuschelrekonstruktion hat Joseph aber offenbar weniger gute Erfahrungen mit dem Material gemacht als bei der Sattelnasenkorrektur. Im Zusammenhang mit der Beschreibung des operativen Vorgehens zum Aufbau einer Ohrmuschel gibt Joseph aber Auskunft über die Präparation der Elfenbeinimplantate: „Das am besten von einem Elfenbeinschnitzer hergestellte Ohrgerüst wird mit Bohrlöchern versehen, damit seine Stellung sicher fixiert und sein ohnehin schon geringes Gewicht noch weiter verringert wird" (Seite 723).

Nicht entnommen werden kann dem Josephschen Lehrbuch, woher das Elfenbein bezogen wurde. Natvig (7) zitiert jedoch Safian (9) als Zeugen dafür, daß das Elfenbein als reichlicher "Abfall" aus einer Fabrik stammte, in der Klaviertasten hergestellt wurden. Safian selbst hat sich in der gleichen Fabrik anläßlich eines Besuches bei Joseph „für den Rest seines Lebens“ mit Elfenbeinrohmaterial für Implantationszwecke versorgt (Natvig, 1982). Daß Joseph ein Faktotum auf Müllplätze und zu Altwarenhändlern zu schicken pflegte, um alte Klaviere aufzutreiben, deren Elfenbeintasten er dann zu Implantaten umfunktionierte, ist wohl der Legende zuzurechnen. An dieser Stelle sei auch erwähnt, daß die immer wieder geäußerte Meinung, Jacques Joseph sei durch eigene Hand oder eines gewaltsamen Todes gestorben, nicht haltbar ist (Reintanz, 1985).

Joseph kann als einer derjenigen gelten, die die Plastische Chirurgie nicht nur operationstechnisch gefördert, sondern sie auch als ärztliche Aufgabe begründet haben (Reintanz, 1985). Außerdem hat er, ohne im klassischen Sinne je Klinikleiter oder Hochschullehrer gewesen zu sein, eine Schule begründet, die in der ganzen Welt weiterlebt - wenn auch seine letzten Elfenbeinspäne bald nicht mehr existieren werden.

\section{Literatur}

1 Bab, M.: Úber die Sterilisation des Elfenbeins. Zentralbl. f. Chir. 26 (1924)

2 Eitner, E.: Über Sattelnasenkorekturen. Dtsch. med. Wschr. 31 (1915)

3 Hauben, D. J.: Jacques Joseph (1865-1934). Laryng. Rhinol. Otol. $62(1983) 56$

4 Hellmich, S.: Operativ wesentliche Gesichtspunkte bei Spanimplantationen der Nase. Z. Laryng. Rhinol. 512 (1972) 298

5 Hellmich, S.: Materialbedingte Gefahren der korrektiven Rhinoplastik. Arch. Oto-Rhino-Laryngol. 223 (1979) 336

6 Joseph, J.: Nasenplastik und sonstige Gesichtsplastik nebst einem Anhang über Mammaplastik und einige weitere Operationen aus dem Gebiete der äußeren Körperplastik. Ein Atlas und Lehrbuch; Verlag Curt Kabitzsch, Leipzig 1931

7 Natvig, P.: Jacques Joseph - Surgical Sculptor. W. B. Saunders (Philadelphia etc.) 1982

8 Reintanz, G.; 80 Jahre intranasale Rhinoplastik. - Zum 50. Todestag von Jacques Joseph. HNO Prax. (Leipzig) 10 (1985) 67

9 Safian, J.: Personal recollections of Professor Jacques Joseph. In: Plast. Reconstr. Surg. 46 (1970) 175

10 Staindl, O.: Zur Therapie der Sattelnase. Laryng. Rhinol. Otol. 62 (1983) 348

\section{Univ.-Prof. Dr. Otto Staindl}

Hals-Nasen-Ohren-Abteilung der Landeskrankenanstalten Salzburg

Müllner Hauptstraße 48

A-5020 Salzburg

Austria

Prof. Dr. Sigurd Hellmich

Hals-Nasen-Ohren-Klinik

St. Johannes Hospital

Johannesstr. 9-15

D-4600 Dortmund

Privatdoz. Dr. Alexander Berghaus

Universitätsklinikum Steglitz

Hals-Nasen-Ohren-Klinik

Hindenburgdamm 300

D-1000 Berlin 45 